\title{
Single-incision surgery for gynecomastia using TriPort: A case report
}

\author{
JIAN LIU, YONG HAN, KAI CHENG, XIAO-HONG WANG, FENGLI GUO and ZHEN-LIN YANG
}

\begin{abstract}
Department of Thyroid and Breast Surgery, Binzhou Medical University Hospital, Binzhou, Shandong 256603, P.R. China
\end{abstract}
Received November 9, 2017; Accepted April 26, 2018

DOI: $10.3892 /$ etm.2018.6224

\begin{abstract}
Gynecomastia is a common benign condition of the male breast, frequently observed in newborns, adolescents and the elderly. Surgical excision remains one of the most effective methods for the management of patients with gynecomastia, particularly those with fibrosis or a course of $>1$ year. The present study describes two cases of patients with gynecomastia and presents a novel method of treatment, combining endoscopic surgery and liposuction using a TriPort, which may effectively remove breast tissue and shorten hospitalization time compared with a direct excision method.
\end{abstract}

\section{Introduction}

Gynecomastia is a common benign condition of the male breast, most frequently observed in newborns, adolescents and the elderly, and it may be associated with discomfort and mental stress (1). The pathogenesis of this condition is multifactorial and in most cases idiopathic (2). Prevalence rates are $60-90 \%$ in newborns, $50-60 \%$ in adolescents and $70 \%$ in males aged 50-69 years (1).

Disease progression has several stages. Asymptomatic males with long-standing breast enlargement do not usually require treatment other than therapy. In males who suffer from pain or embarrassment, treatment is guided by the cause of gynecomastia and patient expectations (3). It is ideal for medicinal treatments to be applied during the proliferation phase of gynecomastia (4). However, if gynecomastia has been present for $>1$ year, it would not be expected to regress substantially, either spontaneously or with medicinal therapy, due to the presence of fibrosis (5). Under these circumstances, surgical excision to remove tissue is the only effective method.

Correspondence to: Professor Zhen-Lin Yang, Department of Thyroid and Breast Surgery, Binzhou Medical University Hospital, 661 Huanghe 2nd Road, Binzhou, Shandong 256603, P.R. China E-mail: jrwktg@163.com

Key words: gynecomastia, single incision, endoscopic plastic surgery, liposuction
Surgical management of gynecomastia includes four general approaches: Excision of breast tissue, liposuction and skin resection, alone or in combination. Liposuction may be used to treat pseudo gynecomastia, as fat deposition is a major concern in such cases (6). Liposuction and excision may be considered when the breast is composed primarily of fibrotic tissue (7). If there is excessive skin, skin resection may also be considered (1). The periareolar incision technique is effective, but endoscopic techniques are associated with a better cosmetic outcome $(1,5,8)$. The present study reports two cases of patients with gynecomastia, who were managed considering cosmetic outcome and efficacy using a TriPort (Fig. 1), which combines endoscopic surgery and liposuction.

\section{Patients and methods}

Case 1. A 51-year-old male exhibited a body mass index of 17.4. According to Simon's classification (9), the right breast had exhibited grade II gynecomastia for 6 years and the left breast had exhibited grade I gynecomastia for 3 years. Following treatment with antiestrogens for 15 months, no breast reduction was observed. Liver function tests and serum creatinine were normal. All endocrinological findings were normal, with the exception of luteinizing hormone levels 17.63 IU/l (Normal range: 1.7-8.6 IU/1). A brain magnetic resonance imaging (MRI) scan was performed, revealing no abnormal findings. On breast ultrasonography, breast tissue echoes were identified, with a range of $4.5 \times 0.8 \times 1.1 \mathrm{~cm}$ on the right and $2.3 \times 0.5 \times 2.2 \mathrm{~cm}$ on the left breast. On physical examination, a rubbery mound of tissue was palpated bilaterally, which was concentric with the nipple-areolar complex. Thus, the patient was diagnosed with gynecomastia according to Braunstein GD's diagnostic criteria (5). The patient requested an endoscopic resection in his right breast and open resection in the left, with periareolar incisions and underwent surgical treatment.

Case 2. A 48-year-old man (body mass index, 17.4) exhibited grade II gynecomastia of the right breast, according to Simon's classification (9) for 6 months. On breast ultrasonography, breast tissue echoes in the right breast were identified with a range of $3.3 \times 2.5 \times 0.8 \mathrm{~cm}$. Liver function tests and serum creatinine were normal. All endocrinological findings were within the normal range, with the exception of prolactin level, which was increased by 2 -fold compared with the normal value (632.90 IU/1). A brain MRI was recommended, but the patient 
refused. The patient underwent direct excision of the left breast tissue 4 years previously. The patient requested single-incision surgery for the right breast.

Surgical technique. Prior to procedure, modalities of the reconstruction were discussed by surgeon and patient. Open and closed procedures were explained and risks of scarring were discussed. Patients were admitted to the Binzhou Medical University Hospital (Binzhou, China) in April 2017 (case 1) and May 2017 (case 2). Informed consent and ethics approval were obtained respectively from patients and the Ethics Committee of Binzhou Medical University (Binzhou, China).

For single-incision surgery for gynecomastia, preoperative markings were made on the patient to ensure precise demarcation of the operative area (Fig. 2). Under general anesthesia, the patient was placed in a supine position, with the arms abducted to $90^{\circ}$ and fixed. A single skin incision was performed along the mid-axillary line in order to hide the scar when the arms are resting at the sides. Lipolysis solution $(200 \mathrm{ml}$ sterile purified water, $200 \mathrm{ml}$ physiological saline, $30 \mathrm{ml} 5 \% \mathrm{NaHCO}$, $20 \mathrm{ml} 2 \%$ lidocaine and $1 \mathrm{ml} 0.1 \%$ adrenaline; total solution volume of $451 \mathrm{ml})(10,11)$ was injected into the subcutaneous and retromammary spaces, and liposuction was performed after $15 \mathrm{~min}$. The volume of liposuction was $204 \mathrm{ml}$ and $182 \mathrm{ml}$ (case 2), as determined by the volume of the aspirator cylinder subtracted by $451 \mathrm{ml}$ lipolysis solution. When liposuction was completed, only the Cooper ligaments remained between breast gland and skin. The sleeve pedestal was inserted in the incision by separating the surrounding subcutaneous tissue. The pedestal was then connected with the TriPort (HTKD Medical; Beijing HangTian KaDi Technology R\&D Institute, Beijing, China; product model, HK-FDDC-4FX). To establish the working space, $\mathrm{CO}_{2}$ was insufflated and inflation pressure was maintained at $8 \mathrm{mmHg}$. The suture line was used to secure nipple and areola. The endoscope, ultrasound knife, assistant clamp and other equipment were fixed on the TriPort (Fig. 3). The four quadrants of the breast, retromammary cellular space, Cooper's ligament and nipple-areolar tissues were separated successively. The excision process was controlled under endoscopic visualization with an ultrasound knife or electrocautery, enabling adequate tissue removal, bleeding control and preservation of the pectoral fascia.

In the left breast of case 1, a direct excision was made through radial incision. Along with the superficial fascia, the fat and mammary gland tissue layers were separated. Furthermore, some gland tissue was left behind the nipple and areola to support the complex. The incision was closed following successful removal of all gland tissue. No equivalent procedure was performed in case 2 .

The total operative time was $60 \mathrm{~min}$ in case 1 and $57 \mathrm{~min}$ in case 2 (mean, $\sim 58 \mathrm{~min}$ ). A drain and gauze compressing the breast surface were used to prevent postoperative bleeding. To achieve a more satisfying cosmetic result, intradermal absorbable sutures were used to close the incision (Fig. 4).

\section{Results}

Patients were discharged on the third (case 1) and second (case 2) postoperative day, following postoperative pathology to confirm gynecomastia diagnosis. All patients

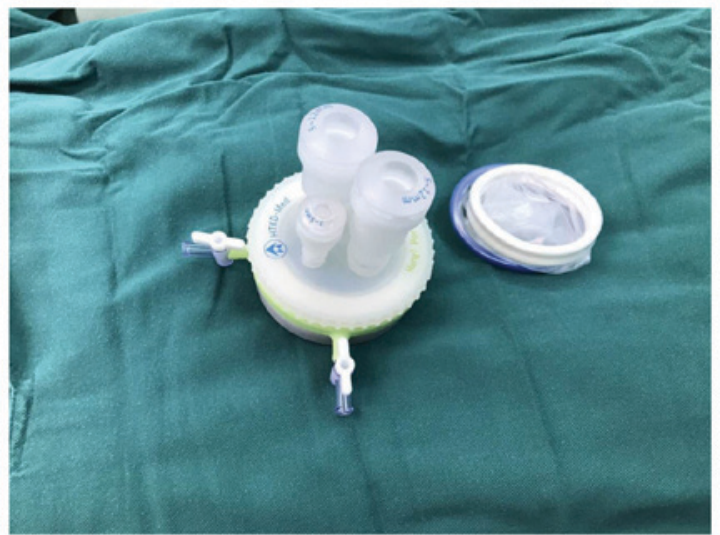

Figure 1. TriPort used for surgery.

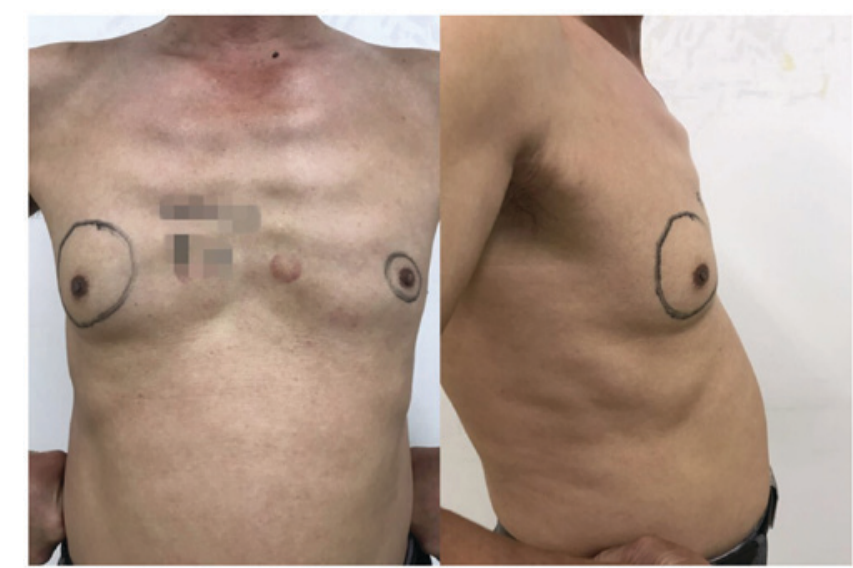

Figure 2. Preoperative markings on the patient; the entire breast was outlined to mark the operative area.

were satisfied with the cosmetic result and short hospitalization time. Less postoperative bleeding $(\sim 110 \mathrm{ml}$ for case 1 and $100 \mathrm{ml}$ for case 2 during hospitalization) was observed compared with the direct excision method, as excision combined with endoscopic visualization using an ultrasound knife and electrocautery achieved hemostasis. Drain and gauze were removed, 5 days following surgery and there were no postoperative complications. Single incisions were completely covered by the arms, while the left periareolar incision in case 1 was more obvious (Fig. 5). There were no recurrences or complications during 5 months of follow-up.

\section{Discussion}

Gynecomastia is a common cause of discomfort and embarrassment for men. Surgical excision remains one of the most effective methods for gynecomastia management, particularly in patients with fibrosis or with a disease course of $>1$ year (7). Previously, minimally invasive techniques, including power-assisted and ultrasound-assisted liposuction, have been a popular approach due to their satisfactory cosmetic outcome (12-15). However, interest is now focused on minimally invasive surgery to minimize external scars (14). Our ultimate goals are minimal incision, better cosmetic outcome, efficacy and reproducibility. 

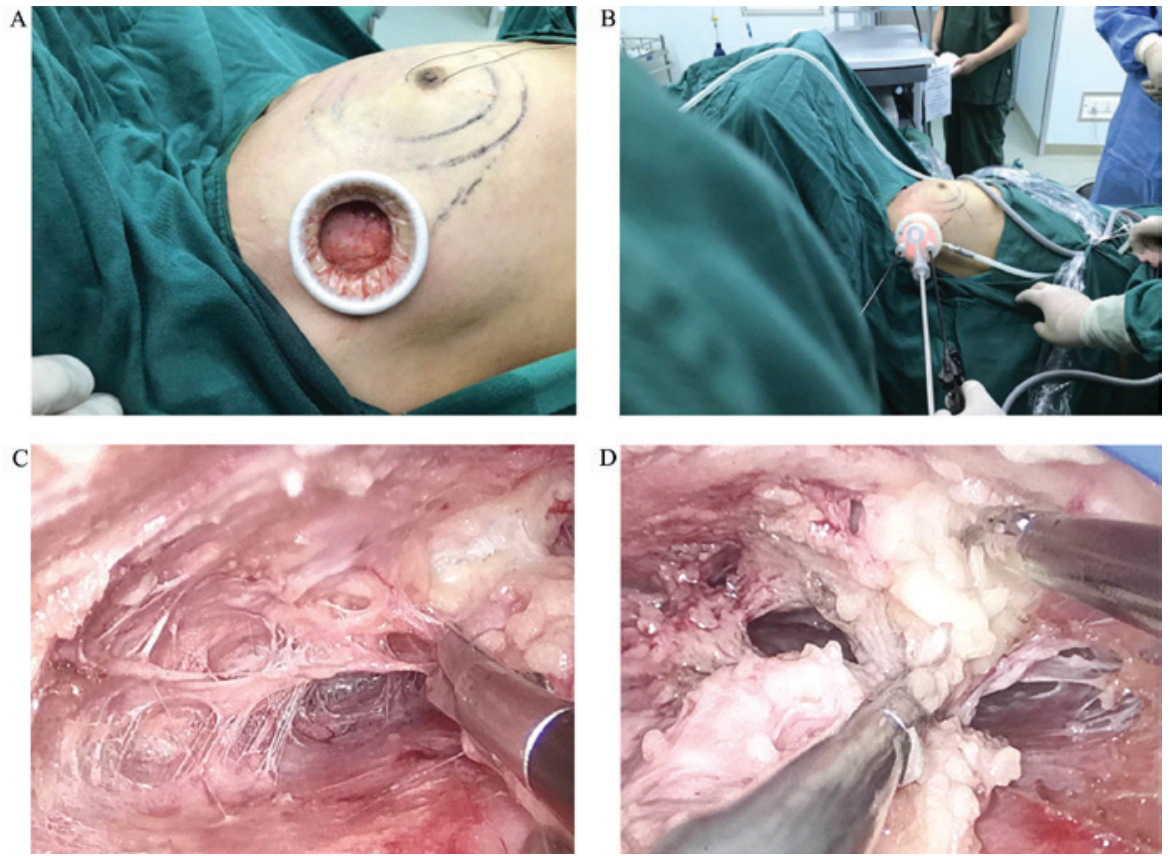

Figure 3. The operative processes highlighting: (A) The sleeve pedestal, (B) the TriPort, (C) separation of Cooper's ligament and (D) nipple and areola.

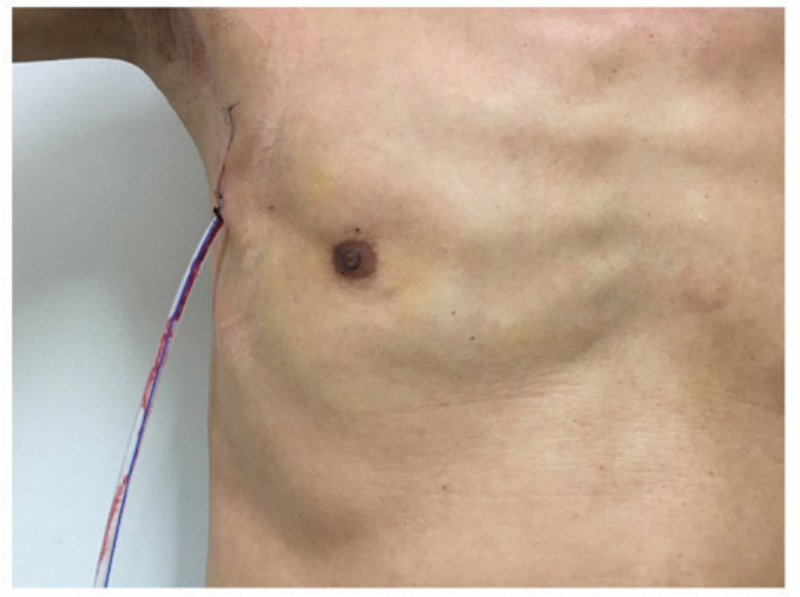

Figure 4. The drain used with the patients and the intradermal absorbable suture used to close the incision.

Jarrar et al (13) used a single 15-mm incision during surgery of gynecomastia. The authors were experienced in endoscopy and were able to achieve a good cosmetic outcome. However, a longer time was required for resecting and extracting tissues and if extracted tissues were larger or of a tougher consistency, the use of scissors or graspers and diathermy may not have been the optimal approach. The size of the incision allowed for the use of an endoscope during surgery. The amplification provided by an endoscope clearly visualized the operating field on the monitor screen, allowing for improved view compared with previous single incision procedures. In addition, the amplification of an endoscope allows the surgeon to identify and cauterize minor bleeds, thereby reducing postoperative bleeding and shortening hospitalization time. Increased surgery length may lead to hypothermia and increase the neurotoxicity of the narcotic agent (16). Balance between incision length and final cosmetic outcome is important during gynecomastia surgery. Zhu and Huang (6)

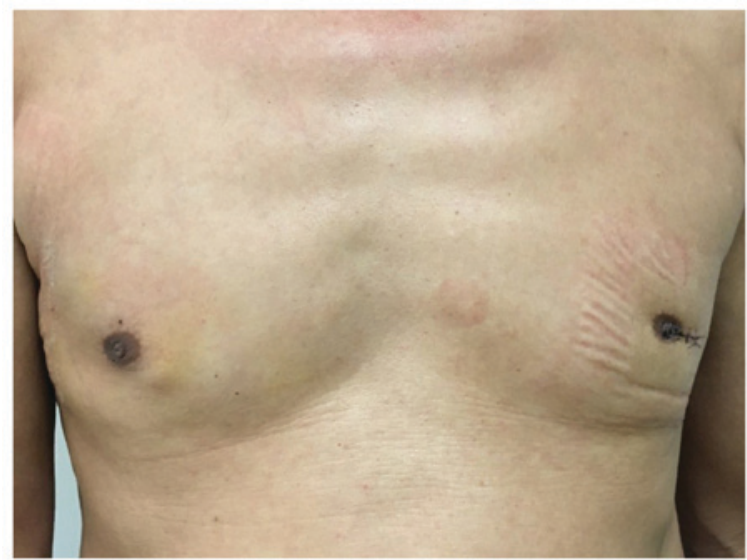

Figure 5. Patient chest following drain and gauze removal on the third postoperative day, who received a single incision on his right breast and a direct excision on his left breast.

reported the use of three incisions to introduce an endoscope. The whole excision process was performed under endoscopic visualization, enabling adequate removal, good bleeding control and preservation of the pectoral fascia. However, the final incision was enlarged to extract excised tissue, which prolonged the procedure. The final incision was longer compared with the single incision performed in the current study, and tissue injury was more extensive.

Recently, Chang and Lee (14) reported an increasing interest in minimally invasive surgery with an emphasis on minimizing scars. The authors demonstrated that liposuction was associated with minimizing incisional scars, but the procedure was less effective in removing glandular tissue. Therefore, direct excision of glandular tissues remained irreplaceable. The present study hypothesized that patients may prefer surgery that enables control under endoscopic visualization in combination with a smaller, but adequate, incision. 
In the current study a TriPort was applied during surgery, which has previously been used in laparoscopic surgery $(17,18)$. The TriPort enables completion of gynecomastia surgery through a single incision, combining endoscopy and liposuction. This TriPort-based method enabled good control of endoscopic and operating instruments, separation of different tissues and hemostasis throughout surgery, and it may shorten operative time. It was previously reported that the mean operative time was $82 \mathrm{~min}$ (range, 65-100 $\mathrm{min}$ ) compared with 58 min observed in the current study (10). Due to different trocars on the TriPort, there is no interference with different tractors, due to the stability provided by TriPort, under endoscopic view, achieving a result of better control of these tractors comparable with that of three-incisional surgery. The excision process was controlled by endoscopic visualization, enabling adequate tissue removal, good bleeding control and preservation of the pectoral fascia. Benefits, including a positive postoperative recovery, less postoperative bleeding, a decreased incidence of complications and shortening of hospitalization period, were not reported in previous single-incision procedures $(13,19)$. Furthermore, the inner diameter of the TriPort was sufficient to extract excised tissue integrally, decreasing the operative time and avoiding residual tissue left following the procedure. Various types of TriPort are available with inner diameters ranging from 50 to $120 \mathrm{~mm}$, which have the potential to remove varying amounts of breast tissue. The tractors on the TriPort provide the option of using other equipment if necessary, achieving a larger surgical space compared with conventional minimally invasive methods. The height of the TriPort is adjustable, ranging from 40 to $200 \mathrm{~mm}$, which may be useful during surgery of obese patients. In addition, extraction of excised tissue en bloc shortens the duration under anesthesia. Therefore, combination of liposuction and endoscopic resection may be an effective method for removing mammary tissue, accompanied by a favorable cosmetic result, decreased hospitalization time compared with direct excision methods and adequate control of bleeding.

To the best of our knowledge, this is the first report describing the use of TriPort, which combines endoscopic surgery and liposuction, in the management of gynecomastia. Single-incision endoscopic surgery through the TriPort may be an effective and safe way for the management of gynecomastia, as it ensures an aesthetic result and complete removal of the glandular tissue. Further studies are required to allow a systematic evaluation of this method.

\section{Acknowledgements}

Not applicable.

\section{Funding}

This study was supported by grants from the National Natural Science Foundation of China (grant nos. 30973932 and 81173601), the Natural Science Foundation of Shandong Province (grant nos. ZR2017LH072, ZR2017MH033 and ZR2014HQ020), the Projects of Binzhou Technology Development Program (grant no. 2015ZC0301), the Scientific Research Staring Foundation of Binzhou Medical University (grant nos. BY2014KYQD36 and BY2014KJ36) and the
Science and Technology Program of Universities in Shandong Province (grant no. J15LL51).

\section{Availability of data and materials}

All data generated or analysed during this study are included in this published article.

\section{Authors' contributions}

JL, KC and YH wrote the manuscript and performed surgery; XW and ZY designed the present study; and FG and JL were responsible for patient follow-up and data collection. All authors read and approved the final manuscript.

\section{Ethics approval and consent to participate}

The present study was approved by the was obtained from Ethics Committee of Binzhou Medical University (Binzhou, China; approval no. 2018-002-01). Written informed consent was obtained from patients prior to enrolment.

\section{Consent for publication}

The patient provided written informed consent for the publication of any associated data and accompanying images.

\section{Competing interests}

The authors declare that they have no competing interests.

\section{References}

1. Sansone A, Romanelli F, Sansone M, Lenzi A and Di Luigi L: Gynecomastia and hormones. Endocrine 55: 37-44, 2017.

2. Rahmani S, Turton P, Shaaban A and Dall B: Overview of gynecomastia in the modern era and the Leeds Gynaecomastia Investigation algorithm. Breast J 17: 246-255, 2011.

3. Carlson HE: Approach to the patient with gynecomastia. J Clin Endocrinol Metab 96: 15-21, 2011.

4. Cao H, Yang ZX, Sun YH, Wu HR and Jiang GQ: Endoscopic subcutaneous mastectomy: A novel and effective treatment for gynecomastia. Exp Ther Med 5: 1683-1686, 2013.

5. Braunstein GD: Clinical practice. Gynecomastia. N Engl J Med 357: 1229-1237, 2007.

6. Zhu J and Huang J: Surgical management of gynecomastia under endoscope. J Laparoendosc Adv Surg Tech A 18: 433-437, 2008.

7. Dickson G: Gynecomastia. Am Fam Physician 85: 716-722, 2012.

8. Waltho D, Hatchell A and Thoma A: Gynecomastia classification for surgical management: A systematic review and novel classification system. Plast Reconstr Surg 139: 638e-648e, 2017.

9. Simon BE, Hoffman S and Kahn S: Classification and surgical correction of gynecomastia. Plast Reconstr Surg 51: 48-52, 1973.

10. Fan L, Yang X, Zhang Y and Jiang J: Endoscopic subcutaneous mastectomy for the treatment of gynecomastia: A report of 65 cases. Surg Laparosc Endosc Percutan Tech 19: e85-e90, 2009.

11. Shi F, Huang Z, Yu J, Zhang P, Deng J, Zou L, Zhang C and Luo Y: Immediate liposuction could shorten the time for endoscopic axillary lymphadenectomy in breast cancer patients. World J Surg Oncol 15: 35, 2017.

12. Brown RH, Chang DK, Siy R and Friedman J: Trends in the Surgical Correction of Gynecomastia. Semin Plast Surg 29: 122-130, 2015.

13. Jarrar G, Peel A, Fahmy R, Deol H, Salih V and Mostafa A: Single incision endoscopic surgery for gynaecomastia. J Plast Reconstr Aesthet Surg 64: e231-e236, 2011.

14. Chang HP and Lee DW: Discussion: Surgical management of gynecomastia: Subcutaneous mastectomy and liposuction. Aesthetic Plast Surg 41: 985-986, 2017. 
15. Kisu I, Banno K, Kobayashi Y, Ono A, Masuda K, Ueki A, Nomura H, Hirasawa A, Abe T, Kouyama K, et al: Flexible hysteroscopy with narrow band imaging (NBI) for endoscopic diagnosis of malignant endometrial lesions. Int J Oncol 38: 613-618, 2011.

16. Jevtovic-Todorovic V, Absalom AR, Blomgren K, Brambrink A Crosby G, Culley DJ, Fiskum G, Giffard RG, Herold KF, Loepke AW, et al: Anaesthetic neurotoxicity and neuroplasticity: An expert group report and statement based on the BJA Salzburg Seminar. Br J Anaesth 111: 143-151, 2013.

17. Erhart D and Pohnán R: 55 laparoscopic cholecystectomies using single incision laparoscopic surgery-initial experience. Rozhl Chir 90: 361-364, 2011 (In Czech).
18. Pagano D, Echeverri GJ, Gridelli B, Spada M, Botrugno I and Bartoccelli C: Natural orifice transumbilical cholecystectomy using a tri-port trocar and conventional instruments. J Am Coll Surg 210: 1013-1014, 2010.

19. Mentz HA, Ruiz-Razura A, Newall G, Patronella CK and Miniel LA: Correction of gynecomastia through a single puncture incision. Aesthetic Plast Surg 31: 244-249, 2007.

(i) (2) This work is licensed under a Creative Common

cc) Attribution-NonCommercial-NoDerivatives 4.0 International (CC BY-NC-ND 4.0) License. 